Article

\title{
On Superconductivity of One-Dimensional Channel with Strong Electron-Electron Interaction
}

\author{
Vasily V. Afonin * and Serge V. Gantsevich * \\ Ioffe Physical-Technical Institute of the Russian Academy of Sciences, 194021 St.Petersburg, Russia \\ * Correspondence: vasili.afonin@mail.ioffe.ru (V.V.A.); sergei.elur@mail.ioffe.ru (S.V.G.)
}

Received: 21 April 2019; Accepted: 21 June 2019; Published: 29 June 2019

\begin{abstract}
We study the ground state of a one-dimensional channel with strong attractive electron-electron interactions at low temperatures. In spite of the fact that, at low temperatures, the ground state of one-dimensional attracting electrons is a state with a macroscopically large number of cooperons, the resulting superconducting phase has a number of significant differences. Namely, the order parameter (which should appear in the superconducting phase according to Landau's phenomenological theory) turns out to be zero. However, elastic impurities implanted in a one-dimensional channel will not lead to dissipation of the supercurrent associated with the condensate movement as a whole.
\end{abstract}

Keywords: superconductivity; one-dimensional electrons

PACS: 73.63.-b; 73.23.-b; 71.10.Pm

\section{Introduction, Problem Statement and Qualitative Discussion of the Results}

In connection with the development of nanotechnologies, which allow for the production of one-dimensional ballistic channels, interest in the theory describing these objects was revived. Another reason for traditionally stimulating theoretical work in this area is that exactly solvable one-dimensional models (see, for example, the papers Luttinger [1], Schwinger [2,3]) gave hope to understand, at the qualitative level, the phenomena occurring in a higher dimension systems. For example, the Schwinger model, for a long time, has been the only field theory to discuss the confinement problem. It should be said that, over a long time, the Luttinger model (one-dimensional interacting electrons without backscattering) was considered a purely theoretical problem too. It gave a number of interesting (from the theory point of view) results. Later, it served as the basis for considering problems related with quasi-one-dimensional problems. However, at the beginning of this century, the first experimental works on quantum wires appeared. One could hope that electrons occupy only the lowest level of transverse quantization $[4,5]$. As a result, the electronic transport in the objects should have been described by a one-dimensional model. It stimulated works related to the calculation of the conductance in the one-dimensional channel, the influence of elastic impurities implanted into the channel [6,7], and the effects associated with the screening of electron-electron interactions by a three-dimensional environment.

In this paper, we will discuss the other question. We will show that (as was to be expected) the ground state of the Luttinger model with a strong electron-electron attraction is a state with a long-range order. Using direct calculation, we will make sure that, in the case of single-component electrons, it contains a macroscopically large number of Cooper pairs (correlated states acting as Bose-like particles). For the multicomponent system, correlated states consist of more complex correlated complexes: fours for two-component fermions (electrons with spin), eights for conducting nanotubes (four-component fermions), etc. This is a phenomenon known in relativistic theories as the 
$\mathrm{t}^{\prime}$ Hooft principle. According to it, the interaction of $n$-component fermions leads to an appearance in $\mid$ in $>$ and $\mid$ out $>$ states the correlated complexes consisting of the maximum possible fermions number permitted by the Pauli principle [8]. In our problem, this means that, for two-component fermions, the correlated complexes consist of two electrons with opposite spins moving right and the same electrons moving left (see Equation (17)). We will call all such complexes cooperons. They always consist of an even number of electrons, and their total spin is zero. From the statistics view point, such objects look like extensive bosons. Therefore, the long-range order arising at the system has a purely statistical nature.

Before discussing the detailed form of the ground state in the one-dimensional case, we briefly discuss the questions of the physical picture: the three-dimensional superconductivity that is important for the one-dimension case. The fundamental property of what we now call the phase with broken symmetry is the "rigidity of the ground-state wave function". It was understood at the early stages of superconductivity theory development (F.London [9]). After understanding instability of the normal metal ground state (Fermi sphere, $\mid F>$ ) relative to infinitely small attractions between electrons, one made arguments in favor of the existence of correlated objects consisting of two electrons with opposite momenta behaving like extensive boson particles in the new ground state (Cooper's pairs [10]). It was the basis for the creation of the consistent theory of superconductivity.

At a qualitative level, the connection between the rigidity of the ground state wave function and the existence of a macroscopic bosons number (i.e., the boson number increase with an increase the system volume, L) was understood, apparently, by R.P. Feynman. In essence, it is based on the normalization factor $\sqrt{N+1}$, arising from the action of the boson creation operator on $N$-bosons wave function (let the state correspond to the movement of all Cooper pairs in one direction-the supercurrent). Let us ask ourselves the following question: What is the probability of finding a boson at another state elastically scattered by an impurity? The ratio of the probabilities (leaves in the $N$-bosons state or scatters to the empty one) is about $1 / N$ [11] (Bose-Einstein principle). If $1 / N \sim 1 / L^{\beta}$, $(0<\beta \leq 1)$, then this relationship is vanishingly small. This shows that the coherence (rigidity) of the ground state requires only a macroscopically large number of Bose particles in the state and not a finite density of pairs. The finite density of Cooper pairs, $\beta=1$, corresponds to the usual second-order phase transition: $\beta<1$ is the Kosterlitz-Thoules-Berezinsky (BKT) phase [12-14].

In fact, this picture needs to clarify the symmetry properties of the scattering, which can transfer the boson to an empty state. Symmetry considerations are extremely important for second-order phase transitions. It was pointed out in Landau phenomenological theory $[15,16]$. Its starting point was the introduction of an order parameter, i.e., a quantity that would have to be zero due to the symmetry of the Hamiltonian. However, it turns out to be nonzero due to the fact that the symmetry of the ground state below the transition point turns out to be lower than the symmetry of the Hamiltonian. Let us discuss what this means in terms of quantum mechanics. The appearance of the nonzero order parameter signifies that a quantum mechanical operator connected with this quantity has the nonzero matrix element $<\Omega|A| \Omega>$ despite the fact that this contradicts the Hamiltonian symmetry (here, $\mid \Omega>$ is the ground state wave function). The invariance of the Hamiltonian with respect to any transformation means the existence of the conservation law and, corresponding to it, eigenvalues and wave functions $\mid \Omega>_{n}$. At the same time, the operator $\hat{A}$ has no diagonal matrix elements in the basis. Therefore, the existence of a nonzero order parameter $<\Omega|\hat{A}| \Omega>$ requires that the ground state wave function should be a package $\left|\Omega>=\sum_{\triangle n} C_{n}\right| \Omega>_{n}$. In turn, this suggests that many states $\mid \Omega>_{n}$ with different quantum numbers should be degenerate in energy. Exact energy degeneration of the states is needed for a temperature $(T)$ equal to zero. For finite temperatures, the packet can have finite breadth with the level interval of the order of $T_{d}$. We will call it the degeneration temperature (it should be calculated in a microscopic theory). In such a case, the transition between states with different quantum numbers is possible only for $T>T_{d}$, and at a low temperature region $T \ll T_{d}$, the existence of a nonzero order parameter is impossible (this requirement is not important for three-dimensional superconductors, where the phase transition temperature $\left(T_{c}\right)$ is determined by the constants of the 
theory and the distance between the levels is determined by the size sample. Therefore, at the limit $L \rightarrow \infty T_{d} \rightarrow 0$, while $T_{c}$ remains finite. Therefore, for a three-dimensional sample, the question is irrelevant, though the case of extremely low temperatures may be an exclusion. However, as we will see later, it is important in a one-dimensional case).

In the case of three-dimensional superconductivity, the ground state ceases to be invariant with respect to the gauge transformation $(\psi \rightarrow \exp (i \Lambda) \psi)$. The Hamiltonian invariance with respect to the gauge transformation leads to the conservation of electric charge. Therefore, in the superconductivity problem under $\mid \Omega>_{n}$, one should understand states with a fixed value of electric charge and that the operator $\hat{A}$ is an operator of the observed value changing the charge of a state. In particular, the violation of the gauge invariance of the theory results in the fact that the charged particles fall out to the condensate. They can consist of two electrons or two holes with opposite momenta. In the usually discussed temperature region, above $T_{d}$ but below $T_{c}$, states with different charges have the same energy. As a result, the ground state wave function is non-invariant with respect to the gauge transformation, and one has a nonzero order parameter.

Another matter is the low-temperature region $T \ll T_{d}$. Here, the ground state is non-degenerate in charge, i.e., an order parameter turns out to be zero. Moreover, if the ground state has a charge equal to zero (usually, it is the lowest energy state), then in this region, there is no global symmetry breaking. In this case, the ground-state wave function has the same symmetry as the Hamiltonian despite the fact that it consists of Cooper pairs and the wave function of each pair is non-invariant with respect to the gauge transformation. However, the presence of impurities in this case does not result in resistance appearance due to the factor $1 / N \sim 1 / L^{\beta}$ in the scattering probability. It is clear that our statement about suppression in the cooperon scattering does not apply to the weak $e-e$ interaction case $(\beta \ll 1)$. It is exactly the region where a renormalization group approach works (see Reference [17] and the references there within).

Returning to properties of the scattering (which is suppressed by many Bose particles that fall out to condensate), it should be invariant with respect to broken symmetry. Otherwise, scattering (it will be strong in a one-dimension case due to $e-e$ interaction renormalization) leads to the level shift and can break up the whole package $\mid \Omega>$. The electrons scattering on elastic impurities is invariant with respect to the gauge transformation. Therefore, this scattering will be suppressed by the macroscopic number of Cooper pairs existing in the ground state.

In the next section, we will calculate the ground state wave function in the Luttinger model with a sufficiently strong attraction [18]. We will show that, at $T=0$, the ground state of a 1-D channel contains a macroscopically large cooperons number. However, the number of correlated complexes consisting of electrons and holes is equal to each other. Thus, the ground state has an electric charge equal to zero and the wave function of the ground state is invariant with respect to the gauge transformation. It makes the existence of the order parameter impossible (note, we are talking not about the density of the order parameter but about its total value. Normally, the latter is proportional to the sample volume). The phase with a global violation of gauge invariance under these conditions is not realized because, in the case of a strong attractive $e-e$ interaction, $T_{d} \gg T_{c}$. The small value of the phase transition temperature is easy to understand, taking into account that, at gap-superconductor, the transition temperature is of the order of normal excitations gap (careful consideration of normal excitations problem is given in Reference [19]). The spectrum of these excitations is well-known and, for the one-component electrons, is $v_{\mathcal{c}}\left|p_{n}\right|$ (here, $v_{\mathcal{C}}\left(p_{n}\right)=v_{f} \sqrt{1+V_{0}\left(p_{n}\right) / \pi v_{f}}, v_{f}$ is the Fermi velocity, and $p_{n}$ is the electron momentum equals to $2 \pi n / L$. The quantity $V_{0}\left(p_{n}\right)$ is the $e-e$ interaction "potential", negative in the problem. We use the system units $\hbar=1)$. Thus, we see that the gap in the excitation spectrum is of the order of $v_{c} / L$. If the length of a one-dimensional channel is of the order of a micron and the electron concentration is about of metallic, one can give the order of magnitude estimation for transition temperature: $v_{c} / L \sim 1^{o} \mathrm{~K} \cdot\left(v_{c} / v_{f}\right)$ while $T_{d} \sim v_{f} / L$ (in fact, it is the only remaining quantity with the dimension of energy and is proportional to $1 / L$ ). The specifics of one-dimensional superconductivity is in a small critical temperature in comparison with the energy 
of degeneracy. Nevertheless, the absence of a global symmetry breaking at temperatures lower than the degeneration temperature does not prevent the existence of a permanent supercurrent (the motion of the condensate as a whole). In this sense, it is possible to speak of a phase transition to the superconducting state in a one-dimensional channel.

\section{Calculation of the Ground State Wave Function}

Our task is to calculate the ground state wave function. In principle, one can find it by solving the Schrödinger equation. However, due to a strong electron-electron interaction, the equation actually has an infinite number of spatial variables and one has no effective method for solving this equation.

Another object that explicitly contains all information about the system is the well-known evolution operator. In the Schrödinger representation (dependence on time is transferred to the wave functions), it can be represented as

$$
S(\tau)=\sum_{m, n}|n><n| \exp (-i \hat{H} \tau)|m><m| .
$$

The evolution operator expresses the development over a finite time $\tau$ as an exact (including interaction) initial state $<m \mid$ to all possible final states $\mid n>$ (these states have yet to be calculated). By "summing up" over all states, we mean the enumeration of all initial and final states, and the indices $m$ and $n$ are complete description of this state. In our case, complete description of the state reduces to specifying the particles number and their quantum numbers. We assume that the wave functions of the system at the initial time $t=0$ (final $t=\tau$ ) are expressed in terms of the annihilation (creation) operators of the right (left) electrons (holes), $\hat{a}(\hat{b})_{R, L}$. They are determined according to the following:

$$
\begin{gathered}
\hat{\Psi}_{R, L}(x)=\int_{0}^{\infty} \frac{d p}{2 \pi}\left(\exp ( \pm i p x) \hat{a}_{R, L}(p)+\exp (\mp i p x) \hat{b}_{R, L}^{+}(p)\right)= \\
=\hat{a}_{R, L}(x)+\hat{b}_{R, L}^{+}(x),
\end{gathered}
$$

and $H$ is the Hamiltonian of the system. In the case of one-component electrons, it is as follows:

$$
\hat{H}=\int d x\left[\hat{\Psi}_{R}^{\dagger}(x) v_{f}\left(-i \partial_{x}\right) \hat{\Psi}_{R}(x)+\hat{\Psi}_{L}^{\dagger}(x) v_{f} i \partial_{x} \hat{\Psi}_{L}(x)\right]+\int d x d y \varrho(x) V_{0}(x-y) \varrho(y) .
$$

Here, $\varrho$ is the electron density equal to $\varrho=\varrho_{R}+\varrho_{L}$. We will count all momenta $(p)$ from Fermi one $\left(p_{f}\right)$. In addition, we will use periodic boundary conditions, i.e., strictly speaking, we should consider the momentum as discrete. However, if the condition $p_{f} L \gg 1$ is fulfilled, in most calculationsm the sum can be replaced by an integral (as it is written in Equation (2)). The quantities $\Psi_{R, L}$ are the wave functions of right and left electrons. It can be determined through the complete electron wave functions according to the following:

$$
\hat{\psi}(x)=\exp \left(i p_{f} x\right) \hat{\Psi}_{R}(x)+\exp \left(-i p_{f} x\right) \hat{\Psi}_{L}(x)
$$

Note that, after transition to the "complex time" $\tau \rightarrow-i / T$, the evolution operator becomes the usual density matrix. Recall that, by the indices " $m$ " and " $n$ " in this density matrix, one needs to understand not only the quantum numbers of an electron but also the electrons number in a given state.

The expression for the evolution operator can be written as a functional integral:

$$
S(\tau)=\int_{(\bar{\Psi}, \Psi)} \mathcal{D} \Psi \mathcal{D} \bar{\Psi} \exp (\mathcal{S})(\bar{\Psi}, \Psi) .
$$

Here, $\bar{\Psi}$ and $\Psi$ are the electron fields (Grassmann variables) and $\mathcal{S}$ is the action. Dependence of the evolution integral on $\tau$ arises from the fact that the integration over time is performed not on the 
infinite interval $(-\infty, \infty)$ but on the interval $(0, \tau)$. However, the main difference in the Equation (3) from the ordinary Feynman partition function consists in the fact that the fields $(\bar{\Psi}, \Psi)$ do not tend to zero at the ends of the time integration domain but satisfy the following boundary conditions:

At $t \rightarrow+0$,

$$
\begin{aligned}
& \Psi_{R, L}(x, t)=a_{R, L}(x)+\text { arbitrary negative frequency part } \\
& \bar{\Psi}_{R, L}(x, t)=b_{R, L}(x)+\text { arbitrary negative frequency part }
\end{aligned}
$$

At $t \rightarrow \tau-0$,

$$
\begin{aligned}
& \Psi_{R, L}(x, t)=b_{R, L}^{\dagger}(x)+\text { arbitrary positive frequency part } \\
& \bar{\Psi}_{R, L}(x, t)=a_{R, L}^{\dagger}(x)+\text { arbitrary positive frequency part }
\end{aligned}
$$

In these boundary conditions, arbitrary positive (negative) frequency parts arise because, after acting on the Fermi sphere $(\mid F>$ is the vacuum state of our theory), they give zero. Note that the exact states (that we have to find yet) in this representation have the form $\phi\left(x_{1}, x_{2}, \cdots\right) \hat{a}_{R, L}^{\dagger}\left(x_{1}\right) \hat{b}_{R, L}^{\dagger}\left(x_{2}\right) \cdots$ $\mid F>$. The boundary conditions dramatically complicate the wave function calculation in comparison with the Green function one (the latter satisfies the zero boundary conditions). In addition, summation over all exact initial and final states in the expression for the evolution Operator in Equation (1), in essence, means that the matrix element is the sum of all n-particle Green functions. As we will see, the ground state wave functions can be analytically calculated only for a sufficiently strong $e-e$ interaction.

Now, let us consider the initial many-body state $(\mid F>)$ over which the creation and annihilation operators are defined. As usual, the operators $\hat{\Psi}_{R, L}^{\dagger}$ are defined over the empty state $\mid 0>$ whereas the operators $\hat{a}_{R, L}^{\dagger}(x)$ and $\hat{b}_{R, L}^{+}(x)$ are defined over the filled Fermi sphere. Moreover, we suppose that our system is neutral as a whole. Therefore, we should introduce some sort of positive charge background ("jelly model") and hence redefine the electric charge of the states. In the case the vacuum, $\mid F>$ is electrically neutral state. Cooper pairs $\hat{\Psi}_{R}^{+} \hat{\Psi}_{L}^{+}\left|0>; \hat{a}_{R}^{+}(x) \hat{a}_{L}^{\dagger}(x)\right| F>$ are the states with the charge $2 e$, while the pair $\hat{b}_{L}^{+}\left(x^{\prime}\right) \hat{b}_{R}^{\dagger}\left(x^{\prime}\right) \mid F>$ has the charge $-2 e$. Therefore, a two-cooperons state $\hat{a}_{L}^{\dagger}\left(x^{\prime}\right) \hat{a}_{R}^{\dagger}\left(x^{\prime}\right) \hat{b}_{L}^{\dagger}\left(y^{\prime}\right) \hat{b}_{R}^{\dagger}\left(y^{\prime}\right) \mid F>$ is neutral. The charge redefined in such a way is directly connected with gauge symmetry, which is usually broken during the superconductor transition. To avoid misunderstandings, we note that the whole electric charge of the entire system, as always, remains. In fact, its conservation is guaranteed by the time-independence normalizing factor of the electron's total wave function (or, in other words, the theory is unitary), unlike changing electric charge of one state. The last simply means a redistribution of the charge between all states (or with a reservoir connected to the sample).

We begin consideration from the limit $T \rightarrow 0$. For this case, one should transform $\tau \rightarrow-i / T$. The evolution operator for the Luttinger model with any $e-e$ interaction was calculated in Reference [20]. However, the very cumbersome result was analyzed there only for repulsive electron potentials. Our attention at that time was focused on the existence of the chiral phase. The phase was new to the solid state physics. It is connected with the chiral symmetry violation $\left(\hat{\Psi}_{R, L} \rightarrow \exp ( \pm i \Lambda) \hat{\Psi}_{R, L}\right)$, and the condensate is created by exciton-like neutral pairs $\hat{a}_{R}^{\dagger}(x) \hat{b}_{L}^{\dagger}(x)$.

Now, we will consider the attraction interaction. Here, we restrict ourselves to discuss the case point-like $e-e$ interaction (Gorkov's model) and equilibrium electron system. We will not reproduce a calculation of the functional integral of Equation (3) in the case of attractive $e-e$ electrons interaction. Here, we limit ourselves by indicating the reason why the analytical expression for the evolution operator allows us to write an expression for the ground-state wave function (at least in the form of an infinite series). In the following, using the general expressions for the ground state wave function obtained earlier in Reference [20], we will analyze it for the attractive electrons.

The possibility to obtain an expression for $\mid \Omega>$ out of the evolution operator is based on the fact that the transition matrix element from the initial state $<m \mid$ to the final one $\mid n>$ factorizes (it is represented as a product of two functions each of which depend only on coordinates particles at initial 
(final) states). Therefore, it can be explicitly represented as $\left|\Omega_{n}\right\rangle \cdot\left\langle\Omega_{m}\right|$. Later, everything will depend on how effectively one can sum the perturbation series for the wave function $\mid \Omega_{n}>$.

At $T=0$, the ground state wave function can be written as an infinite series:

$$
\begin{array}{r}
\mid \Omega>=\sum_{n=0}^{\infty} \frac{1}{n !}\left[\int \frac{d x d x^{\prime}}{\pi i} \frac{\hat{a}_{R}^{\dagger}(x) \hat{b}_{R}^{\dagger}\left(x^{\prime}\right)}{x^{\prime}-x-i \delta}+\right. \\
\left.+\int \frac{d y d y^{\prime}}{2 \pi i} \frac{\hat{a}_{L}^{\dagger}(y) \hat{b}_{L}^{\dagger}\left(y^{\prime}\right)}{y-y^{\prime}-i \delta}\right]^{n} \exp \mathcal{S}_{c n f}\left(x, x^{\prime}, . ., y, y^{\prime}, . .\right) \mid F>,
\end{array}
$$

In this expression the pre-exponential factor actually is the most general type of wave function with zero electric charge, and the whole really informative part is contained in the form of configuration action $\left(\mathcal{S}_{c n f}\left(x, x^{\prime}, . ., y, y^{\prime}, ..\right)\right)$. It is different for each term of the series:

$$
\mathcal{S}_{c n f}\left(x \ldots, x^{\prime} \ldots, y \ldots, y^{\prime} \ldots . .\right)=\frac{\pi}{L} \alpha \sum_{n \neq 0} \frac{1}{\left|p_{n}\right|} \mathcal{R}_{f}\left(-p_{n}\right) \mathcal{R}_{f}\left(p_{n}\right),
$$

where $\alpha=\left[1-v_{c} / v_{f}\right] /\left[1+v_{c} / v_{f}\right]$ and

$$
\begin{gathered}
\mathcal{R}_{f}(p)=\sum_{x . . ; x^{\prime} . . ; y . . ; y^{\prime} \ldots} \theta(p)\left[\exp (i p x)+\ldots-\exp \left(i p x^{\prime}\right)-\ldots\right]+ \\
\theta(-p)\left[\exp (i p y)+\ldots-\exp \left(i p y^{\prime}\right)-\ldots\right]
\end{gathered}
$$

Here and below, we will denote the letter $x$ as the coordinates of the right electrons, $x^{\prime}-$ holes, $^{\prime}$ and, respectively, $y$ and $y^{\prime}$ coordinates of the left electrons and holes that appear in each term sums (Equation (5)). "Summation" occurs over all particles of which the creation operators are in the pre-exponential factor in the expression for the ground-state wave function. In these expressions, we can already move from the discrete to the continuous spectrum $(L \rightarrow \infty)$. The finite size of the system for our problem is only important until the moment of neglecting of the terms exponentially small in the parameters $2 \pi n v_{(f, c)} / L T$. As a result, the channel length is included only in the parameters of the theory related to temperatures, and all calculations, in fact, are done as for an infinite sample.

In order to transform Equation (5), one recalls that, according to the logarithm connectedness theorem (in statistical physics, it is known as the first Mayer theorem [21]), the wave function can be represented as an exponent of the connected diagrams sum [22], i.e., connected terms of Equation (5). Disconnected diagrams, which also exist in $|\Omega\rangle$, are generated by the power terms of the exponent decomposition. This means that the expression for the ground state wave function can be represented in an explicit analytical form in the case when the number of connected diagrams is small.

The task is extremely simplified in the case of a strong $e-e$ interaction. As we will see, in this limit, the condensate consists of a macroscopically large number of point-like Cooper pairs. They do not interact with each other. In the case of point-like $e-e$ interactions, the scattering of the pairs is possible if the two identical electrons (that make up the Cooper pairs) are at the same point. This is excluded by the Pauli principle. At a weaker $e-e$ interaction, the Cooper pair acquires a finite radius and the Pauli principle ceases to suppress the scattering of cooperons. However, from the expression for $v_{c}$, it is seen that, with a very large constant of the attractive $e-e$ interaction, the excitation spectrum becomes imaginary, i.e., the system collapses. Physically, this means that it is necessary to modify the potential of the $e-e$ attraction, adding a hard repulsive core to it. It makes calculations much more complicated. Instead, we restrict ourselves to the case of a relatively weak interaction $\left(\left|V_{0}\right| / \pi v_{f} \leq 1\right)$ when the spectrum of the excitations still remains real.

In order to understand the specifics of our problem, we first consider the maximally strong attraction $\left|V_{0}\right| / \pi v_{f}=1$, i.e., $v_{c}=0$. We will see that this limit describes a ground state with a nonzero concentration of noninteracting Cooper pairs and a total electric charge equals to zero. Next, we will 
take into account small corrections in the configuration action, proportional to $v_{\mathcal{C}}$, and make sure that they transform this state into a Kosterlitz-Thoules-Berezinsky phase [10,12,13].

Consider the first few terms of the series in Equation (5). Because of the $\theta$ functions included in $\mathcal{R}_{f}(p)$, in the wave function, there are nonzero only terms containing right and left electrons simultaneously and the number of electron and hole operators have to be the same. The simplest of these states is $\hat{a}_{R}^{\dagger}(x) \hat{b}_{R}^{\dagger}\left(x^{\prime}\right) \hat{a}_{L}^{\dagger}(y) \hat{b}_{L}^{\dagger}\left(y^{\prime}\right) \mid F>$. The contribution of this configuration to the action at $v_{c}=0$ is

$$
\mathcal{S}_{c n f}\left(x \ldots, x^{\prime} \ldots, y \ldots, y^{\prime} \ldots\right)=-\ln \frac{(x-y+i \delta)\left(x^{\prime}-y^{\prime}+i \delta\right)}{\left(x^{\prime}-y+i \delta\right)\left(x-y^{\prime}+i \delta\right)}
$$

As a result, in the expression for the wave function of the ground state, we obtain the following:

$$
\int \frac{d x d x^{\prime} d y d y^{\prime}}{(2 \pi i)^{2}} \frac{\hat{a}_{R}^{\dagger}(x) \hat{b}_{R}^{+}\left(x^{\prime}\right) \hat{a}_{L}^{\dagger}(y) \hat{b}_{L}^{+}\left(y^{\prime}\right)}{\left(x^{\prime}-x-i \delta\right)\left(y-y^{\prime}-i \delta\right)} \frac{\left(x^{\prime}-y+i \delta\right)\left(x-y^{\prime}+i \delta\right)}{(x-y+i \delta)\left(x^{\prime}-y^{\prime}+i \delta\right)}
$$

Further analysis of a contribution will be based on the analytical properties of the creation and annihilation operators. As follows from Equation (2), the operators $a_{L}^{\dagger}(y)$ and $\hat{b}_{L}^{\dagger}\left(y^{\prime}\right)$ are analytical in the upper half-plane and $\hat{a}_{R}^{\dagger}(x) \hat{b}_{R}^{\dagger}\left(x^{\prime}\right)$ is analytical in the lower one. Thus, the integrals are determined by the pole residue at the points $x^{\prime}=y^{\prime}-i \delta ; x=y-i \delta$ and become the product of two disconnected diagrams describing two noninteracting Cooper pairs.

$$
\int d x \hat{a}_{R}^{\dagger}(x) \hat{a}_{L}^{\dagger}(x) \int d x^{\prime} \hat{b}_{L}^{\dagger}\left(x^{\prime}\right) \hat{b}_{R}^{\dagger}\left(x^{\prime}\right) \mid F>
$$

Let us make sure that all other diagrams that are not reducible to the power of that one are equal to zero. Consider, for example, the six-fermion contribution to Equation (5):

$$
\frac{\hat{a}_{R}^{\dagger}(x) \hat{b}_{R}^{\dagger}\left(x^{\prime}\right) \hat{a}_{R}^{\dagger}\left(x_{1}\right) \hat{b}_{R}^{\dagger}\left(x_{1}^{\prime}\right) \hat{a}_{L}^{\dagger}(y) \hat{b}_{L}^{\dagger}\left(y^{\prime}\right)}{\left(x^{\prime}-x-i \delta\right)\left(x_{1}^{\prime}-x_{1}-i \delta\right)\left(y-y^{\prime}-i \delta\right)} \frac{\left(x^{\prime}-y+i \delta\right)\left(x_{1}-y^{\prime}+i \delta\right)\left(x_{1}^{\prime}-y+i \delta\right)\left(x-y^{\prime}+i \delta\right)}{(x-y+i \delta)\left(x_{1}-y+i \delta\right)\left(x^{\prime}-y^{\prime}+i \delta\right)\left(x_{1}^{\prime}-y^{\prime}+i \delta\right)}
$$

This integral will be determined by the poles $x \rightarrow y \rightarrow x_{1} ; x^{\prime} \rightarrow y^{\prime} \rightarrow x_{1}^{\prime}$ in the lower half-plane, and we get the configuration $\hat{a}_{R}^{\dagger}(x) \hat{a}_{R}^{\dagger}(x) \hat{a}_{L}^{\dagger}(x) \cdot \hat{b}_{R}^{\dagger}\left(x^{\prime}\right) \hat{b}_{R}^{\dagger}\left(x^{\prime}\right) \hat{b}_{L}^{\dagger}\left(x^{\prime}\right)$. It is equal to zero according to the Pauli principle. It can be verified that the remaining diagrams reduce to these two cases. Therefore, one has only two connected diagrams. However, applying the logarithm connectedness theorem, one should keep in mind that, in our case, only states with a total electric charge equal to zero exist and that the remaining states should be omitted. We denote as $P(Q=0)$, the projector onto this state is as follows:

$$
\left|\Omega>=N_{0} P(Q=0) \exp \left[\int d x \hat{a}_{R}^{\dagger}(x) \hat{a}_{L}^{\dagger}(x)+\int d x^{\prime} \hat{b}_{L}^{\dagger}\left(x^{\prime}\right) \hat{b}_{R}^{+}\left(x^{\prime}\right)\right]\right| F>,
$$

Here, the $N_{0}$ is the normalization factor, and it can be calculated. Thus, in this approximation, we have obtained the condensate composed of the non-interacting Cooper pairs in one state: $\hat{a}_{R}^{\dagger}(x) \hat{a}_{L}^{\dagger}(x) ; \hat{b}_{L}^{\dagger}\left(x^{\prime}\right) \hat{b}_{R}^{\dagger}\left(x^{\prime}\right)$ (with a nonzero density and full charge equal to zero). We note once again the peculiarity of the obtained ground state. On the one hand, there is no global violation of gauge invariance (each configuration contains an equal number of electrons and holes, and the phase of the gauge transformation vanish). As a result, the long range order parameter (one can enter it, for example, like this: $<\Omega\left|\hat{a}_{R}^{\dagger}(x) \hat{a}_{L}^{\dagger}(x)\right| \Omega>$ ) is equal to zero due to the electric charge conservation law. On the other hand, one has a macroscopically large number of Bose-like particles in one state, i.e., the system turns out to be completely statistically correlated (all electrons are pairing. In the ground state, there are only Cooper pairs and their number is macroscopically large). In this case, the Bose-Einstein principle guarantees the impossibility of scattering pairs on elastic impurities added to the system under consideration. 
We now discuss how this picture changes when small corrections proportional to $v_{c} / v_{f}$ are taken into account (that is, $\alpha$ is close to 1). In order to show that, in this case, one has a condensate that consists of the macroscopically large number of Cooper pairs, we should extract the correlated complexes from the whole wave function (Equation (5)). For this, one should present the four-particles state that we discussed earlier,

$$
\int \frac{d x d x^{\prime} d y d y^{\prime}}{(2 \pi i)^{2}} \frac{\hat{a}_{R}^{\dagger}(x) \hat{b}_{R}^{+}\left(x^{\prime}\right) \hat{a}_{L}^{\dagger}(y) \hat{b}_{L}^{+}\left(y^{\prime}\right)}{\left(x^{\prime}-x-i \delta\right)\left(y-y^{\prime}-i \delta\right)}\left[\frac{\left(x^{\prime}-y+i \delta\right)\left(x-y^{\prime}+i \delta\right)}{(x-y+i \delta)\left(x^{\prime}-y^{\prime}+i \delta\right)}\right]^{\alpha} \mid F>
$$

as a two correlated complexes product (their sizes should be small compared to the sample size). They are separated from each other by a large distance (of the order of $L$ ). It can be seen from this expression that the probability of finding the right electron near the left hole $\left(\left|x-y^{\prime}\right| \rightarrow 0\right)$ is suppressed by the interaction and that the probability of finding the left electron near the right one $(|x-y| \rightarrow 0)$ increases. Therefore, the contribution from the correlated complexes consisting of right and left electrons is determined by the cut at the points $x-y+i \delta=0 ; x^{\prime}-y^{\prime}+i \delta=0$. Moreover, we should assume that $\left|y-x^{\prime}\right| \sim\left|x-y^{\prime}\right| \sim R \rightarrow \infty$. Therefore, the contribution from the first cut is proportional to

$$
\left(1-e^{2 \pi i \alpha}\right) \int_{-\infty}^{x} \frac{d y}{2 \pi i} \frac{\hat{a}_{L}^{\dagger}(y)}{(x-y)^{\alpha}} \frac{\left(x^{\prime}-y\right)^{\alpha}}{\left(y-y^{\prime}\right)} \sim \hat{a}_{L}^{\dagger}(x)\left(\frac{d}{R}\right)^{1-\alpha} .
$$

Here, we have taken into account that the integrand converges well for $y \rightarrow \infty$ (due to the boundary conditions, the fields $\hat{a}_{L}^{\dagger}(y)$ are zero on the sample's surface). In this case, the contribution from the complex is determined by the upper limit of integration and should be cut off at $|y-x| \sim d$ (here, $d$ is the ultraviolet cutoff. It is of the order of the channel thickness: On this scale, the $e-e$ interaction becomes three-dimensional and one-dimensional effects are suppressed). Similarly, the "integration" over $x^{\prime}$ is done (later, this procedure will be done more carefully for multicomponent fermions). As a result, the whole four-particles contribution describes a state with two interacting complexes (two Cooper pairs $\hat{a}_{R}^{\dagger}(x) \hat{a}_{L}^{\dagger}(x)$ and $\hat{b}_{L}^{+}(y) \hat{b}_{R}^{+}$) are separated from each other by a large distance (of the order of the channel length):

$$
\left|\Omega_{c p l}>=\int d x d y\left(\frac{d}{|x-y|}\right)^{2(1-\alpha)} \hat{a}_{R}^{\dagger}(x) \hat{a}_{L}^{\dagger}(x) \hat{b}_{L}^{\dagger}(y) \hat{b}_{R}^{\dagger}(y)\right| F>.
$$

Thus, instead of a disconnected diagram (9), at $v_{c} \neq 0$, we have a united complex consisting of two interacting Cooper pairs. From this calculation, it becomes clear that the connected diagrams number is infinite and that the theory does not have a parameter that allows one to discard a large number of interacting many-body complexes. It does not allow one to write the wave function of the ground state in a simple form. However, the general form of $S_{c n f}$ (see Equation (7)) is valid for any value of $v_{\mathcal{C}} \neq 0$. It guaranties us that all these terms contain the same number of electron and hole operators (i.e., their electric charge is zero). Consequently, as in the term we considered (Equation (11)), all correlated complexes consist of Cooper pairs interacting with each other and located at a distance of the order of $L$. Therefore, for any $v_{c}$, the ground state will consist of an infinite number of Cooper pairs and, for $T=0$, will have an electric charge equal to zero.

In fact, even the index in the expression of Equation (11) is calculated up to a factor about unity. We calculated it in an approximation in which the cooperons interact only "directly" (their interaction with each other through the other pairs in the intermediate states is not taken into account). However, it is not difficult to correct. For that, it is necessary to renormalize the interaction of the two labeled pairs, taking into account all other cooperons. To do this, it suffices to calculate the two-particle correlator $<\Psi_{R}(x) \Psi_{R}^{\dagger}(y) \Psi_{L}(x) \Psi_{L}^{\dagger}(y)>=<G_{R}(x-y) G_{L}(x-y)>$ at $|x-y|=R \rightarrow L$ and to compare the degree of $R$. Probably, direct renormalization of the interaction in the wave function is possible. It requires derivation of a closed equation for the renormalized interaction of two Cooper pairs in the absence of a parameter. However, the final answer at the output of this procedure is clear from the 
correlator. In it, the renormalization of the interaction for the two-particles Green function is taken into account exactly. Therefore, we should replace $\alpha$ by

$$
\alpha_{T}=1-\frac{v_{\mathcal{C}}}{v_{f}} .
$$

Note that, for small $v_{\mathcal{c}}, \alpha_{T}$ differs from $\alpha$ only by a factor before $v_{\mathcal{c}} / v_{f}$. However, even a decomposition of the ground state wave function with respect to the interacting pairs number makes it possible to verify that the number of cooperons in the ground state increases with increasing $L$. Indeed, it is clear from Equation (11) that the probability $(Z(R))$ to find two Cooper pairs separated by a distance $R$ can be estimated as $(1 /|R|)^{2\left(1-\alpha_{T}\right)}$. Therefore, the number of Cooper pairs for any $v_{\mathcal{c}} / v_{f}$ can be estimated from

$$
N^{2} \sim \int_{0}^{L} d x d y Z(x-y) \sim L^{2 \alpha_{T}}
$$

and $N$ grows with sample size as

$$
N \sim L^{1-v_{c} / v_{f}} .
$$

As we have already discussed in the Introduction, this fact alone is sufficient to state that, according to the Bose-Einstein principle, a supercurrent in one-dimensional channel does not dissipate due to the scattering of the Cooper pairs on elastic impurities. An exception can be the case of a relatively weak $e-e$ interaction where the factor $1 / N$ is not too small [11].

We now turn to the discussion of the finite temperature region $T_{d} \gg T \gg T_{\mathcal{c}}$. For the theory with a repulsion $e-e$ interaction (where the opposite inequalities are realized), the finite temperatures were carefully studied in Reference [20]. We will not reproduce this derivation for attracting electrons because, first, the value of $T_{c}$ is understandable from general considerations (it was discussed in Introduction). Secondly, the impossibility existence of a phase with a global violation gauge symmetry in this case is visible without computing. Therefore, we confine ourselves to discuss changes in the expression for the condensate wave function of Equation (5) in the temperature region.

Dependence of the ground state wave function occurring from boundary conditions (from the terms with creation and annihilation operators in Equation (2)) can be extracted easily from the entire action. In Reference [20], it is shown that the contribution to the action from the nonzero boundary conditions is equal to

$$
\begin{aligned}
\mathcal{S}_{0}=\sum_{i=R, L} \int d x d x^{\prime} & {\left[b_{i}\left(x^{\prime}\right) G_{i}\left(x^{\prime}, 0 ; x, \epsilon\right) a_{i}(x)+a_{i}^{\dagger}\left(x^{\prime}\right) G_{i}\left(x^{\prime}, \tau ; x, \tau-\epsilon\right) b_{i}^{\dagger}(x)\right.} \\
& \left.-a_{i}^{\dagger}\left(x^{\prime}\right) G_{i}\left(x^{\prime}, \tau ; x, 0\right) a_{i}(x)-b_{i}\left(x^{\prime}\right) G_{i}\left(x^{\prime}, 0 ; x, \tau\right) b_{i}^{\dagger}(x)\right] .
\end{aligned}
$$

Therefore, $\hat{S}(\tau) \sim \exp \left(S_{0}\right)|F><F|$ (in the expression for the ground state wave function $\mid \Omega>$ (Equation (5)), the factor obtained by factorizing this contribution in the density matrix was expanded into the series). To get this expression, we used the Hubbard trick [23] and reduced the $e-e$ interaction problem to the problem of noninteracting electrons placed in a slowly varying external field (later, a result should be averaged over all realizations of the random Hubbard's field). Therefore, $G_{i}\left(x^{\prime}, t^{\prime} ; x, t\right)$ appearing in the equation is the noninteracting electron Green function in the external field $(\epsilon$ in Equation (13) is a infinitely small value). It is well-known that the Green function of an electron in an external field is proportional to the free Green's function, which we should write in the final-size volume:

$$
G_{R, L}^{0}\left(x^{\prime}, t^{\prime} ; x, t\right)=\frac{1}{L} \sum_{n \neq 0} e^{2 \pi i n\left[x-x^{\prime} \mp v_{f}\left(t-t^{\prime}\right)\right] / L} .
$$

In order to present it in a usual pole form the neighboring terms in the sum, it should not change much. This is so, in the first two terms of the action $S_{0}$, the Green function enters at coinciding times. In the third and fourth terms, the time argument is large and, after transition to imaginary time, has the 
exponent index $-2 \pi T_{d} n / T$. Therefore, in the region of interest to us $\left(T_{d} / T \gg 1\right)$, these contributions to the action are exponentially small. That is why they were omitted at $T \rightarrow 0$. Now, we have to ignore them too. The first two terms at $T \rightarrow 0$ have been taken into account. We have seen that they brought about to the states with zero electric charge. In the case of repulsive electrons (where $T_{c} / T_{d} \gg 1$ ), we could consider the region $T_{d} / T \ll 1$. Then, the last two terms brought about the states with nonzero chiral charges.

In the temperature range $T \gg T_{c}$, dramatic changes also occur in the expression for the configuration action arising from the first two terms. The coefficient $\alpha$ in Equation (6) changes. It is replaced by a function proportional to $\left|p_{n}\right| v_{c} / T$. The last leads to the destruction of the logarithmic divergence in the region of small momenta. It makes the existence of a correlated phase impossible. Therefore, $T_{c}$ really is the critical temperature.

An estimation of the phase transition temperature can be understood from another point of view. As it is known, according to the Landau-Mermin-Wagner theorem, the existence of the BKT phase in one-dimensional systems is impossible at a nonzero temperature. The proofs of this statement for different problems were given first in Reference [24,25]. However, this statement relates to infinite samples and is based on the Goldstone theorem, according to which the spontaneous violation of continuous symmetry must be accompanied by the appearance of a massless boson field (an acoustical mode). Such fields at finite temperatures strongly fluctuate the results in the exponential decay of a correlator at large distances. In our case, the massless Goldstone's mode will be the phase of operator $\Psi_{R}^{\dagger}(x) \Psi_{L}^{\dagger}(x)$, while the correlator which characterizes the system is $<\Psi_{R}^{\dagger}(x) \Psi_{L}^{\dagger}(x) \Psi_{L}(y) \Psi_{R}(y)>$. This correlator for an infinite sample can be calculated (see, e.g., Reference [20]). The power-like behaviour of the correlator is valid in the region $|x-y| \ll v_{c} / T$, and it is exponential at the inverse limiting case. For the finite sample at temperatures $T \ll T_{c}=v_{c} / L$ and $|x-y| \sim L$, the exponential asymptotic does not realize inside a one-dimensional channel. Therefore, inside of the sample, the decay of the correlator is power-like and slower as compared to the case of noninteracting electrons. Just because of this, the number of cooperons in a condensate becomes macroscopically large (see Equation (12)). In this case, the phase transition temperature can be obtained as the estimate $\mid x-$ $y \mid \sim L \sim v_{c} / T$. Thus, at a temperature lower than $T_{c}$, the BKT phase can be realized in the finite one-dimensional sample. Actually, we only define more accurately what one should understand under zero temperature in the Landau-Mermin-Wagner theorem.

Just in case, let us discuss our results, taking into account the duality requirement of the theories with attractive and repulsion electrons. It is clearly visible in the boson representation and requires the replacement of $v_{c} / v_{f}<->v_{f} / v_{c}$ (the ratio $v_{f} / v_{c}$ is usually called the Luttinger parameter, $K$ ). According to it, the solution number of a hypothetically solved many-electron Schrödinger equation for these problems has to be the same. At the same time, in the theory with repulsion electrons, there are two correlated phases: with a global violation of chiral symmetry and with a chiral charge equal to zero while, in the case of attraction, there is only one: electrically neutral. Therefore, the question arises whether this fact does not contradict to the duality requirement? One has a new value with the dimension of energy namely the temperature. In fact, we have just seen that, at the level of the Hamiltonian solutions, an oscillating wave function with a nonzero electric charge also exists for attractive electrons, as duality requires (see the last two terms in Equation (13)). Any correlated phase will be destroyed since, at a sufficiently high temperature, the normal excitations begin to emerge in a large number. Therefore, the temperature of $T_{C}$ in the gap-superconductors is determined by a gap in the spectrum of the excitations: $\omega\left(p_{n}\right)=v_{c}\left|p_{n}\right|$. It follows that a distance up to an excited state in the Luttinger model is about $2 \pi v_{c} / L$ for the both types interactions. This value plays role of a gap (it is clear, at least from dimension considerations, that the replacement $v_{\mathcal{C}}->1 / v_{\mathcal{c}}$ in the spectrum of excitations during the transition from one problem to another is impossible). Therefore, a solution with a global symmetry violation is realized in the case of repulsive electrons $\left(v_{f} / L \ll T_{c}\right.$ and terms with the factor $\exp \left(-\left|p_{n}\right| v_{f} / L T\right)$ at the region $T_{d} \ll T \ll T_{c}$ should be considered). However, this 
solution cannot realize in the problem with attracting electrons, since in this case, the destruction of the superconducting phase occurs first.

We now briefly discuss the problem of multicomponent fermions (for repulsive electrons, this problem was discussed in Reference [26]). From a technical point of view, this problem is much more cumbersome. There is no parameter under which the correlated complexes would not dissipate each other. The latter follows from the fact that the Pauli principle does not forbid the interaction of the complex containing the $\hat{a}_{R, \uparrow}^{\dagger}$ particle with the complex having $\hat{a}_{R, \downarrow}^{\dagger}$ even in the case of a point-like $e-e$ interaction (here, $\uparrow, \downarrow$ is a spin index; we will denote it later as $\alpha= \pm 1$ ) (in fact, the scattering of complexes is much stronger because, even in the case of the strongest $e-e$ interaction $\left(v_{c}=0\right)$, the correlated complexes are not point-like). However, in the problem of multicomponent fermions, one can write an analytical expression for the ground state wave functions, then make sure that the ground state of the system contains the macroscopic number of Bose particles, and identify the quantum numbers of this state.

In order not to complicate the discussion, we consider the case when the interaction of all electrons is the same. Then, the spectrum of quasiparticle excitations for all possible states will be also the same and equal to

$$
\omega\left(p_{m}\right)=\left|p_{m}\right| v_{F} \sqrt{1+\frac{\left.n V_{0}\left(p_{m}\right)\right)}{\pi v_{F}}}
$$

Here, $n$ is the fermion components number (we will discuss mainly the case $n=2$ ). The contribution of boundary conditions to the action remains the same (Equation (13)) with one exception: One should add the sum over the spin index $\alpha$ (in our case, each term in $S_{0}$ is diagonal over the spin-index because the Luttinger model contains only a density-density interaction. That is, it does not describe the Kondo effect). This immediately implies that, in the case of multicomponent fermions, only the state with zero charges will be realized (in this case, one more quantum number is added to the electric charge- - the total spin of the state). This follows from the fact that, in $S_{0}$, the first two terms consist of the same number of electron and hole operators (i.e., they are neutral in the sense of any charge) and only the last two terms can lead to configurations with nonzero quantum numbers. The latter will bring into the action the summands smallness $\exp \left(-T_{d} / T_{c}\right)$. Therefore, the superconducting phase exists only in the region $T \ll T_{c} \ll T_{d}$.

We will now discuss the question about the form of correlated complexes in a problem with multicomponent fermions at $v_{c}=0$. The configuration action in this case undergoes a minimal change; the expression in Equation (6) gets the common factor $1 / n$. As we will see, such interaction weakening is compensated by an increase in the number of particles at each correlated complex.

In order to verify this, we first need to discuss the following question: How, in the case of multicomponent electrons, will we see a combination of particle operators is a correlated complex? In the one-component fermions problem and $v_{c}=0$, this question was irrelevant. The infrared divergence of the action led to the appearance of logarithmic terms of Equation (8) in $S_{c n f}$ with a coefficient equal to 1 . This meant the emergence of new poles and the destruction of the poles arising from the free Green function. As a result, the analytical properties of the annihilation operators allowed us to "calculate" the integral, knowing the residue at the appeared pole. After this, the expression entering the wave function of the ground state was factorized, and for $v_{c}=0$, we obtained noninteracting Cooper pairs. Now, the logarithmic terms in the action have a coefficient of $1 / 2$ and cuts appear in the integrand. This makes representing an operator expression in a compact form impossible. From a common point of view, this means the nonlocality of cooperon (even in the strongest interaction case).

In order to present a general expression for the wave function of the ground state as interacting complexes, we should take several steps. First of all, we should take the connected diagram in the expression for the wave function and try to divide it on the connected compact complexes consisting of a smaller number of particles. To do this, we should move apart the complexes in a large distance $(\sim L)$, keeping the distances between the particles inside each complex small. If, in this limit, the expression 
for our contribution to $\mid \Omega>$ is factorized and c-number function in the expression will not tend to zero, then we can consider these complexes as "new particles" (in this case, the probability to find one complex does not depend on the coordinates of the other complex). In other words, we should consider our contribution to $\mid \Omega>$

$$
\int \frac{d x_{+} d y_{+}}{2 \pi i} \ldots \hat{a}_{R,+}^{\dagger}\left(x_{+}\right) \ldots K\left(x_{+}, \ldots, y_{+} \ldots\right) a_{L,+}^{\dagger}\left(y_{+}\right) \ldots
$$

and to look at the behavior of amplitude $K\left(x_{+}, \ldots, y_{+} \ldots\right)$ provided that the coordinate difference inside the group of variables $x$ and $y$ is small $(\ll L)$ while the coordinates difference between the variables $x$ and $y$ is about $L$. If we chose cooperons correctly, then in this limit, $K\left(x_{+}, \ldots, y_{+} \ldots\right) \rightarrow$ $k_{1}\left(x_{+}, \ldots\right) k_{2}\left(y_{+} \ldots\right)$. This means that, as a result of the interaction, two correlated complexes appeared and their contribution to the ground-state wave function is as follows:

$$
\int \frac{d x_{+}}{\sqrt{2 \pi i}} \ldots k_{2}\left(x_{+} \ldots,\right) \hat{a}_{R,+}^{\dagger}\left(x_{+}\right) \ldots \int \frac{d y_{+}}{\sqrt{2 \pi i}} k_{1}\left(y_{+} \ldots, \hat{a}_{L,+}^{\dagger}\left(y_{+}\right) \ldots \mid F>,\right.
$$

while the connected part, $K-k_{1} k_{2}$, is the scattering amplitude (it tends to zero when $\left|x_{i}-y_{j}\right| \rightarrow \infty$ ). The amplitude should be taken into account at renormalizing interaction between two complexes (as it was done early when $\alpha$ was replaced by $\alpha_{T}$ at $\mid \Omega_{c p l}>$ ). It is reasonable to leave it in the Hamiltonian for the interaction cooperons and to consider the obtained ground state as the $\mid$ out $>$ state (it is exactly the out-state wave function of the scattering problem). The logarithm connectedness theorem $[21,22]$ ensures that the entire wave function will be represented as an exponent of the sum connected complexes. However, later, we will have to take into account the quantum numbers selection rules (for our case, apply a projector onto the state with charge zero to the wave function).

We will show how this procedure can be implemented in the case of two-component fermions with $v_{c}=0$. Based on previous experience, one would expect that the smallest of the possible complexes are ordinary Cooper pairs. The procedure described above gives the following:

$$
\int d x d y \hat{a}_{R,+}^{\dagger}(x) \hat{a}_{L,-}^{\dagger}(x) \hat{b}_{L,-}^{\dagger}(y) \hat{b}_{R,+}^{+}(y) \frac{d}{|x-y|} \mid F>.
$$

Therefore, their feasible contribution to the $\mid$ out $>$ state is of the order of $d / L$ (moreover, the term does not factorize).

The first complexes correlating at the scale of about $L$ consist of four particles (as it should be expected from the $t^{\prime}$ Hooft principle [8]). They are derived from the term

$$
\frac{\hat{a}_{R,+}^{\dagger}\left(x_{+}\right) \hat{b}_{R,+}^{+}\left(x_{+}^{\prime}\right)}{x_{+}^{\prime}-x_{+}-i \delta} \frac{\hat{a}_{R,-}^{+}\left(x_{-}\right) \hat{b}_{R_{,}-}^{+}\left(x_{-}^{\prime}\right)}{x_{-}^{\prime}-x_{-}-i \delta} \frac{\hat{a}_{L,+}^{\dagger}\left(y_{+}\right) \hat{b}_{L_{,}+}^{+}\left(y_{+}^{\prime}\right)}{y_{+}-y_{+}^{\prime}-i \delta} \frac{a_{L,-}^{+}\left(y_{-}\right) b_{L_{,}-}^{\dagger}\left(y_{-}^{\prime}\right)}{y_{-}-y_{-}^{\prime}-i \delta}
$$

obtained as a decomposition of $e^{S_{0}}$. The amplitude of $K$ resulting from the $e-e$ interaction and valid on all scales equals

$$
\begin{aligned}
K\left(x_{+}, \ldots\right)=\frac{1}{x_{+}^{\prime}-x_{+}-i \delta} & \frac{1}{x_{-}^{\prime}-x_{-}-i \delta} \frac{1}{y_{+}-y_{+}^{\prime}-i \delta} \frac{1}{y_{-}-y_{-}^{\prime}-i \delta} \\
& \times \frac{\sqrt{\prod_{\alpha, \beta}\left(x_{\alpha}^{\prime}-y_{\beta}+i \delta\right)\left(x_{\alpha}-y_{\beta}^{\prime}+i \delta\right)}}{\sqrt{\prod_{\alpha, \beta}\left(x_{\alpha}-y_{\beta}+i \delta\right)\left(x_{\alpha}^{\prime}-y_{\beta}^{\prime}+i \delta\right)}} .
\end{aligned}
$$

We should select correlated complexes from our common operator expression. The new complex appearance is due to the fact that the interaction suppresses the probability to find an electron near a hole (see the pole terms) and increases the probability to find two electrons with opposite 
spins close to each other (or two holes). Therefore, the candidates for cooperons are the states $\hat{a}_{R,+}^{\dagger}\left(x_{+}\right) \hat{a}_{L,-}^{\dagger}\left(y_{-}\right) \hat{a}_{R,-}^{\dagger}\left(x_{-}\right) \hat{a}_{L,+}^{\dagger}\left(y_{+}\right)$and $\hat{b}_{R,+}^{\dagger}\left(x_{+}^{\prime}\right) \hat{b}_{L,-}^{+}\left(y_{-}^{\prime}\right) \hat{b}_{R,-}^{+}\left(x_{-}^{\prime}\right) \hat{b}_{L,+}^{+}\left(y_{+}^{\prime}\right)$. We should assume that the distances between the particles entering each state are close to each other and that the distances between these states are large (of the order of $L$ ). In this case, the amplitude (Equation (16)) factorizes but does not tend to zero. Each particle configuration enters the ground state wave function with the factor $V_{4}$. It depends only on one-complex coordinates and is equal to

$$
V_{4}\left(x_{\alpha}, . .\right)=1 / \sqrt{\prod_{\alpha, \beta= \pm}\left(x_{\alpha}-y_{\beta}+i \delta\right)} .
$$

It is easy to verify that the connected part of the scattering amplitude is not factorized and tends to zero when cooperons move apart at a distance about $L$ :

$$
V_{\text {coll }}\left(x_{\alpha . . .} x_{\alpha}^{\prime} \ldots\right)=K\left(x_{+}, \ldots x_{\alpha}^{\prime} \ldots\right)-V_{4}\left(x_{\alpha}, . .\right) V_{4}\left(x_{\alpha}^{\prime}, . .\right)
$$

Therefore, it should be interpreted as a vertex in the interacting cooperons Hamiltonian. The wave function of the ground state is

$$
\begin{gathered}
\mid \Omega>=N_{0} P(Q=0) \exp \left[\int d x_{+} \ldots V_{4}\left(x_{+}, . .\right) \hat{a}_{R,+}^{+}\left(x_{+}\right) \hat{a}_{L,-}^{+}\left(y_{-}\right) \hat{a}_{R,-}^{+}\left(x_{-}\right) \hat{a}_{L,+}^{+}\left(y_{+}\right)+\right. \\
\left.+\int d x_{+}^{\prime} \ldots V_{4}\left(x_{+, . .}^{\prime}\right) \hat{b}_{R,+}^{+}\left(x_{+}^{\prime}\right) \hat{b}_{L,-}^{+}\left(y_{-}^{\prime}\right) \hat{b}_{R,-}^{+}\left(x_{-}^{\prime}\right) \hat{b}_{L,+}^{+}\left(y_{+}^{\prime}\right)\right] \mid F>.
\end{gathered}
$$

The state is realized when the interaction between cooperons, $V_{\text {coll }}$, is adiabatically turned off at $t \rightarrow(\tau, 0)$, i.e., it is the $\mid$ out $>$ state of the system in the cooperons representation.

The ground state of a strong interacting two-component electrons system is a macroscopically large number of Bose-like strongly correlated cooperons consisting of two right and two left electrons (or holes) with opposite spins. The wave function of each complex is non-invariant with respect to the gauge transformation, but the number of complexes consisting of electrons and holes is always equal to each other. Therefore, there is no global violation of gauge invariance in the system.

As we have just seen, even in the case of the strongest $e-e$ interaction $\left(v_{c}=0\right)$, our task has been reduced to the interacting cooperons system. An analysis of diagrams describing the mutual scattering of cooperons shows that, although their interaction is not weak, the qualitative picture of the phenomenon will not change (it also happened in the case of one-component fermions at $v_{c} \neq 0$; see Equation (11)). All that is possible to write in this case is the infinite decomposition of the exact wave function cooperons by their number. This expansion looks rather cumbersome and, from a physical point of view, does not provide new information.

Accounting the finiteness of $v_{\mathcal{C}}$ in our case leads to the replacement of the powers in the expressions for $V_{4}$ and $V_{\text {coll }}$. The degrees $1 / 2$ are replaced by $\alpha / 2=(1 / 2) \cdot\left[1-v_{c} / v_{f}\right] /\left[1+v_{c} / v_{f}\right]$. After replacement, Equation (17) remains correct. It is clear that the case of $v_{c} / v_{f} \rightarrow 1$ is outside of our approach. In this region, the interaction of the cooperons will lead to the destruction of the coherence phase. In this case, it is more rational to use the renormalization group approach formulated in the original electron wave functions representation [17].

The conducting carbon nanotubes give one more example of multicomponent interacting electrons $(n=4)$. According to Reference [27], a conductive nanotube can be consider a cylinder of small radius obtained by gluing a monatomic layer of graphite. If the technology of nanotube production is sufficiently perfect (there is no electron's reflection along the gluing line), then the $e-e$ interaction in the case is described by the Luttinger model with four-component fermions. One can make sure that, in this case, the cooperons will be eight-component correlated complexes.

It remains to discuss the experimental possibility to confirm the form of the ground state in one-dimensional superconducting channels. We have just shown that the distinctive feature of the superconducting ballistic channels is the electroneutrality of the ground state. This means that in the 
one-dimensional case only, simultaneous addition of two Cooper pairs is possible (for the case of the single-component fermions, it is $\hat{a}_{R}^{\dagger} \hat{a}_{L}^{\dagger}$ and $\hat{b}_{R}^{+} \hat{b}_{L}^{\dagger}$ ). Therefore, we believe that the effects associated with coherent tunneling of cooperons are promising for researches.

\section{Conclusions}

In the paper, it was shown that, at a low temperature (less than $2 \pi v_{c} / L$ ), a Luttinger liquid with attraction between $n$-component electrons is a system with a macroscopically large number (i.e., increasing with increasing channel length) of the Bose-like particles (cooperons) in one state. These correlated complexes consist of $2 n$ electrons (or holes). It is the maximum possible number of fermions of which the existence at one point is allowed by the Pauli principle. Although the wave function of each correlated complex is non-invariant with respect to the gauge transformation, the ground-state wave function turns out to be invariant with respect to this transformation. Therefore, the symmetry of the ground state wave function coincides with the symmetry of the Hamiltonian. A global spontaneous violation of gauge invariance does not occur in the system. It takes place because the ground state degeneration temperature in the case of one-dimensional superconductivity turns out to be higher than the temperature of the phase transition to the normal state. As a consequence of this fact, it is impossible to introduce an order parameter in one-dimensional superconductivity. Nevertheless, the presence of the macroscopic number of cooperons in the ground state ensures a long-range order with the suppression of cooperon-impurity scattering and, as the consequence, to the absence of relaxation in the permanent supercurrent in a 1-D channel. Also, the absence of charge degeneration of the ground state (see Equations (10) and (17)) permits adding cooperons only by pairs that conserve the condensate charge.

Author Contributions: Investigation, V.V.A.; Methodology, S.V.G.

Funding: This research received no external funding.

Acknowledgments: We are grateful V.Yu. Petrov for the very useful discussions.

Conflicts of Interest: The authors declare no conflict of interest.

\section{References and Notes}

1. Luttinger, J.M. An Exactly Soluble Model of a Many-Fermion System. J. Math. Phys. 1963, 4, 1154. [CrossRef]

2. Schwinger, J. Gauge Invariance and Mass. Phys. Rev. 1962, 128, 2425-2429. [CrossRef]

3. Kogut, J.; Susskind, L. Quark confinement and the puzzle of the ninth axial-vector current. Phys. Rev. D 1974, 10, 3468-3475. [CrossRef]

4. Bockrath, M.; Cobden, D.H.; Lu, J.; Rinzler, A.G.; Smalley, R.E.; Balents, L.; McEuen, P.L. Luttinger-liquid behaviour in carbon nanotubes. Nature 1999, 397, 598-601. [CrossRef]

5. Lee, J.; Eggert, S.; Kim, H.; Kahng, S.J.; Shinohara, H.; Kuk, Y. Real Space Imaging of One-Dimensional Standing Waves: Direct Evidence for a Luttinger Liquid. Phys. Rev. Lett. 2004, 93, 166403. [CrossRef] [PubMed]

6. Kane, C.L.; Fisher, M.P.A. Transmission through barriers and resonant tunneling in an interacting one-dimensional electron gas. Phys. Rev. B 1992, 46, 15233-15262. [CrossRef] [PubMed]

7. Furusaki, A.; Nagaosa, N. Single-barrier problem and Anderson localization in a one-dimensional interacting electron system. Phys. Rev. B 1993, 47, 4631-4643. [CrossRef] [PubMed]

8. t'Hooft, G. Computation of the quantum effects due to a four-dimensional pseudoparticle. Phys. Rev. D 1976, 14, 3432. [CrossRef]

9. London, F. Superfluids; Dover Press: New York, NY, USA, 1950.

10. Bardeen, J.; Cooper, L.N.; Schrieffer, J.R. Theory of Superconductivity. Phys. Rev. 1957, 108, 1175-1204. [CrossRef] 
11. In order to estimate the ratio let us discuss at first the results obtained for one-component electrons in the case of very strong electron-electron interaction $\left(v_{c}=0\right)$. At this limit the problem has the exact solution and the expression (10) shows that all electrons form pairs and the ground state represents a gas of non-interacting point-like Cooper pairs. From the statistic point of view these pairs manifest themselves as bosons in the same state. Now include the electron-impurity scattering and try to formulate the problem in terms cooperons scattering on impurities. In the case the transition amplitude (due to the impurity scattering) from the state with $N$ cooperons $(<N \mid)$ into an empty state $\mid 0>$ and the amplitude of the system to remain in the state $\mid N>$ differ by a particle number in the final state. So, the first vertex should contains the small quantity $1 / \sqrt{N}$ (it tends to zero in the case of macroscopically large particles number in the ground state). At the decreasing of the electron-electron interaction the pairs begin to interact mutually. Under such condition the ground state calculation has no exact solution even without impurity and does not permit the iteration by the parameter $v_{c} / v_{f}$. In this case a part of cooperons leaves the ground state. However, the expression (12) gives the estimate for a number of pairs remaining in the ground state. In such a case the factor $1 / \sqrt{N}$, having purely combinatorial nature, should be present in the relation discussed. Of course, this argument can be valid only asymptotically by $1 / N$, i.e., we should consider this quantity as the smallest parameter of the theory taking into account the impurity scattering.

12. Berezinsky, V.L. Destruction of Long-range Order in One-dimensional and Two-dimensional Systems having a Continuous Symmetry Group I. Classical Systems. Sov. Phys. JETP 1971, 32, 493-500.

13. Berezinskii, V.L. Destruction of Long-range Order in One-dimensional and Two-dimensional Systems Possessing a Continuous Symmetry Group. II. Quantum Systems. Sov. Phys. JETP 1972, 34, 610.

14. Kosterlitz, J.M.; Thouless, D.J. Ordering, metastability and phase transitions in two-dimensional systems. J. Phys. C Solid State Phys. 1973, 6, 1181-1203. [CrossRef]

15. Landau, L.D. On the theory of phase transitions I. Sov. Phys. JETP 1937, 7, 19-32; [Translation: Phys. Zs. Sowjet, 1937, 11, 26.] [CrossRef]

16. Landau, L.D.; Lifshitz, E.M. Statistical Physics; Butterworth-Heinemann: Oxford, UK, 1986.

17. Giamarchi, T. Quantum Physics in One Dimension; Clarendon Press: Oxford, UK, 2003.

18. Often the Luttinger model is called exactly solvable. This is true, but only in the sense that one can calculate any $n$-particles Green function. Calculating the ground state wave functions is much more complicated procedure. We will see that it can be make analytically only in the limit of very strong e-e interactions and one-component fermions.

19. Afonin, V.V.; Petrov, V.Y. Is the Luttinger Liquid a New State of Matter? Found. Phys. 2010, 40, 190-204. [CrossRef]

20. Afonin, V.V.; Petrov, V.Y. BKT phase in systems of spinless strongly interacting one-dimensional fermions. J. Exp. Theor. Phys. 2008, 107, 542-563. [CrossRef]

21. Uhlenbeck, G.E; Ford, G.W. Lectures in Statistical Mechanics; American Mathematical Society: Providence, RI, USA, 1963.

22. Slavnov, A.A.; Faddeev, L.D. Introduction to Quantum Theory of Gauge Fields; Steklov Math. Inst.: Moscow, Nauka, 1988. [English transl.: Gauge Fields: Introduction to Quantum Theory; Frontiers Phys., 83, Addison-Wesley, Redwood City, Calif. (1991).]

23. Hubbard, J. Calculation of Partition Functions. Phys. Rev. Lett. 1959, 3, 77-78. [CrossRef]

24. Landau, L.D. On the theory of phase transitions II. Sov. Phys. JETP 1937, 7, 627. [translation: Phys. Zs. Sowjet, 1937, 11, 545.]

25. Mermin, N.D.; Wagner, H. Absence of Ferromagnetism or Antiferromagnetism in One- or Two-Dimensional Isotropic Heisenberg Models. Phys. Rev. Lett. 1966, 17, 1133-1136. [CrossRef]

26. Afonin, V.V.; Gurevich, V.L.; Petrov, V.Y. Spontaneous symmetry breaking in a system of strongly interacting multicomponent fermions (electrons with spin and conducting nanotubes). J. Exp. Theor. Phys. 2009, 108, 845-855. [CrossRef]

27. Ajiki, H.; Ando, T. Electronic States of Carbon Nanotubes. J. Phys. Soc. Jpn. 1993, 62, 1255-1266. [CrossRef]

(C) 2019 by the authors. Licensee MDPI, Basel, Switzerland. This article is an open access article distributed under the terms and conditions of the Creative Commons Attribution (CC BY) license (http://creativecommons.org/licenses/by/4.0/). 